\title{
EDITORIAL
}

\section{¿Desde cuándo sentimos?, un recorrido por la evidencia desde la vida intrauterina}

When do we start feelling? a journey through the evidence from intrauterine life perspective

Filiación:

Asociación Costarricense de Alzheimer y Otras Demencias Asociadas-ASCADA. San José, Costa Rica.

Correspondencia: $\square$

Norbel Román Garita. Correo electrónico: drnorbelroman@gmail. com

$---------$

Forma de citar: Román Garita N. ¿Desde cuándo sentimos?, un recorrido por la evidencia desde la vida intrauterina. Rev Ter. 2019;13(2):4-7

Financiamiento:

Ninguno

Conflictos de Interés:

Ninguno

Fecha enviado: 23 marzo del 2019.

Fecha aceptado: 22 junio del 2019.
Norbel Román-Garita $\square$

Hoy nos enfrentamos cada vez mas a retos en el área del conocimiento científico en todos los ámbitos de la vida humana, en sus diferentes etapas del desarrollo; esto en mucho dado por la mayor velocidad en que obtenemos información y también por los avances en temas que parecían ya establecidos que no dejaban a la imaginación la posibilidad de que sucedieran otros aspectos diferentes.

Partiendo desde el conocimiento básico de la biología ya sabemos claramente que la vida humana inicia desde la concepción, siendo desde ese primer momento el inicio de un camino de transformaciones, que en gran parte son evidentes cuando ya en edad adulta podemos observar nuestros cambios y los de otras personas cercanas. Es una realidad contundente, que el proceso de desarrollo humano genera cambios en nuestro aspecto físico, cognitivo, social y en todos los otros campos del desarrollo humano que nos caracterizan e individualizan.

Una de las experiencias más intensas que todos hemos experimentado en nuestras vidas desde muy temprana edad es el dolor físico, que nos permite entre otras razones protegernos de las condiciones de riesgo, anticipar una dolencia y hacernos claramente conscientes de nuestra condición de vulnerabilidad ante una adversidad. Pero también nuestras diferentes modalidades de percepciones de sensibilidad, nos permiten tener una relación con el ambiente y con nosotros mismos, a su vez favorecer el aprendizaje ante las experiencias sensoriales buenas o malas, que terminan estableciendo cambios neuroanatómicos que llamamos memoria, que finalmente se manifiesta en conductas que se reflejan en nuestro diario vivir.

Este viaje por la vida, desde el primer momento hasta la muerte, requiere que tengamos una capacidad de percibir diferentes modalidades de sensibilidad y de dolor como parte necesaria de nuestra característica humana, pero es evidente que deba surgir la pregunta: ¿Qué pasa en la vida intrauterina con respecto al dolor? 
Para tratar de contestar esta pregunta se realizó una búsqueda bibliográfica con apoyo de la Biblioteca Nacional de Salud de Costa Rica (BINASS) con los términos de "dolor fetal" OR "sensación fetal".

De acuerdo al conocimiento básico y a la información obtenidaal día dehoy, necesitamosalgunos requisitos esenciales para poder considerar que podemos sentir dolor o tener la capacidad de percibir algún tipo de sensibilidad en la vida intrauterina, como son receptores de sensibilidad, nervios sensoriales que transmitan, raíces dorsales desarrolladas, una vía espinotalámica en condiciones de buena funcionalidad, una región talámica desarrollada y una corteza cerebral sensorial presente y activa.

Teniendo esto elementos presentes, les invito a un recorrido por la sensibilidad y percepción del dolor desde la vida intrauterina que, aunque invisible a nuestros sentidos y conocimientos tradicionales, nos podría sorprender pues es aún poco explorada y un campo donde la evidencia científica sigue agregando más elementos de confirmación.

Sabemos ahora que nuestra corteza cerebral está desarrollada en el $99 \%$ antes de nacer, lo que nos pone sobre aviso, sobre un ambiente con condiciones aptas para la percepción dolorosa de diferentes modalidades.

Nuestros primeros receptores de sensibilidad en la vida intrauterina se encuentran presentes desde las 7 semanas de gestación, iniciando su ubicación primaria en la región perioral, región de la cara a las 11 semanas, hombros y caderas a las 14 semanas y piel de otros lugares a las 20 semanas ${ }^{1,2}$. De tal manera que en general a las 20 semanas contamos con estructuras físicas de receptores sensoriales capaces de hacernos sentir dolor.

Otra estructura necesaria que nos permite integrar esta informaciones es el tálamo, que es una estructura del diencéfalo con múltiples conexiones y núcleos con diferentes funciones de conectividad, que inicia su diferenciación embriológica tan tempranamente como las 6 semanas y que ya a las 12 semanas tiene fibras ascendentes sensitivas y a las 20 semanas integra conexiones corticales, incluyendo la ínsula a las 15 semanas, sitio donde se desarrolla la experiencia dolorosa. A su vez la corteza ya había logrado iniciar la migración neuronal a las 8 semanas y terminado a las 20 semanas justamente en el tiempo de la conectividad con el tálamo ${ }^{1,2}$.

Pero toda esta evidencia de elementos neuronales no sería suficientes sin tener fibras nerviosa adecuadamente mielinizadas, lo cual se inicia a las 30 semanas y termina incluso después de nacer casi iniciando la edad adulta, sumado al desarrollo de sinapsis que inicia a las 8 semanas y que a las 20 semanas igualmente se tiene un desarrollo suficiente $\mathrm{e}^{1,2}$.

Para las vías dolorosas es importante mencionar que existen de diferentes tipos pero son las llamadas fibras de tipo $C$, sin mielina, las que son especialmente relacionadas a la sensación de dolor y que están presentes más tempranamente en el desarrollo embrionario (entre 8 a 10 semanas) ${ }^{1,2}$, por lo que en este capitulo de requerimientos esta con mayor preparación mas tempranamente en la vida intrauterina.

Sumando todos los datos anteriores es que podemos considerar hasta el día de hoy, que a las 20 semanas post concepción se tienen todas las estructuras necesarias para experimentar el dolor, pero ahora, surge la interrogante si en la vida intrauterina existe la evidencia de respuesta al dolor, o bien el niño o niña por nacer se encuentra en un medio con sustancias inhibidoras del mismo, o bien en un estado de sueño, o bien con sedación natural, como anteriormente se suponía con la evidencia conocida hasta ese momento. 
De acuerdo a la evidencia obtenida en una revisión sobre este tema de literatura publicada entre los años 1990 al 2016, se logró establecer que los neutrotransmisores de tipo neuroinhibidor presentes naturalmente en el feto, no logran un efecto anestésico suficiente como antes se pensaba, por lo que se requiere en caso de anestesia o sedación, por alguna razón clínica, utilizar medios externos en caso procedimientos intrauterino, pues en la segunda mitad del embarazo los neuroinhibidores están en niveles iguales a la madre, quien tiene sensibilidad al dolor. Es por ello que la analgesia y anestesia es mandataria durante la segunda mitad del embarazo, según se establece en algunos consensos de anestesia ${ }^{3-7}$.

Contamos con alguna evidencia para contestar algunas interrogantes sobre la actividad cortical y respuestas del niño o niña por nacer ante estímulos dolorosos, entre ellas la presencia de actividad de electroencefalografía observada en niños nacidos en pretérmino, a las 23 semanas, o bien utilizando magneto encefalografía, también se ha podido corroborar actividad cortical con respuestas evocadas auditivas y visuales fetales tempranas, desde las 26 semanas inclusive. De igual manera se han confirmado respuestas corticales a las 24 semanas luego de punzar el talón o punción venosa durante procedimientos clínicos ${ }^{8,9}$.

Mas recientemente se está utilizando el ultrasonido de 4 dimensiones, que se está convirtiendo en un instrumento clínico para nuevas aplicaciones, entre ellas mejorar el diagnóstico de condiciones intrauterina, análisis de condición clínica, incluso el análisis neuroconductual y de condición de neurodesarrollo en sus diferentes estadios. Incluso, es posible, con esta tecnología, corroborar respuestas físicas y conductuales ante estímulos vibrocusticos que excedan al ruido de fondo $y$ respuesta diferenciada a la vocalización materna.
Se ha podido desarrollar una prueba llamada Kurjak, que permite con el análisis de diferentes variables, conocer el neurodesarrollo y describe observaciones del feto, tales como movimientos de variadas características, desde movimientos bruscos (7 a 8 semanas), movimientos de extremidades (10 semanas) y movimientos complejos (11 semanas) que, de acuerdo a sus análisis, no son casuales o aleatorios ${ }^{10}$. Se ha podido establecer también, un cambio en el conocimiento sobre la actividad fetal, pues se consideraba que la mayoría del tiempo el feto pasaba en un estado inactivo, siendo que permanece en un estado activo de hasta de $59 \%$ de la vida intrauterina, en algunas etapas del desarrollo. Hata ${ }^{10}$, ha podido corroborar movimientos asociados al tacto de la madre sobre la superficie abdominal, observando movimientos de la cabeza y boca, ante la voz materna, se dan también otros movimientos diferenciados, pudiéndose observar estos mismos patrones en la vida extrauterina.

Estamos en los inicios de una nueva forma de ver la vida intrauterina, donde será cada vez más accesible realizar evaluaciones neuroconductuales, ver el desarrollo neurológico con más parámetros clínicos, definir incluso el stress fetal, considerar la posibilidad de detectar dolor fetal y otros elementos, que serán de gran apoyo para los equipos multidisciplinarios en perinatología.

En esta consideración de la evidencia desde la ciencia, hoy tenemos la gran oportunidad de considerar a este ser humano en desarrollo con una dimensión de mayor respeto a sus derechos, incluyendo si su condición llega a ser incompatible con la vida, el apoyo de equipos de cuidados paliativos, en casos de extrema necesidad, pero también nos evidencia una gran oportunidad de explorar nuevas formas de comunicación y estímulo temprano entre otras cosas. 


\section{Referencias Bibliográficas}

1. Bellieni CV, Buonocore G. Is fetal pain a real evidence? J Matern Fetal Neonatal Med. 2012;25(8):1203-1208. doi: $10.3109 / 14767058.2011 .632040$

2. Lee SJ, Ralston HJP, Drey EA, Partridge JC, Rosen MA. Fetal pain: a systematic multidisciplinary review of the evidence. Jama. 2005;294(8):947-954. doi:10.1001/ jama.294.8.947

3. De Tina A, Palanisamy A. General Anesthesia During the Third Trimester: Any Link to Neurocognitive Outcomes? Anesthesiol Clin. 2017;35(1):69-80. doi: 10.1016/j. anclin.2016.09.007

4. Bonnet MP. Sedation and anaesthesia for non-obstetric surgery. Anaesth Crit Care Pain Med. 2016;35:S35-S41. doi: 10.1016/j.accpm.2016.06.009

5. Prabhu M, Bortoletto P, Bateman BT. Perioperative pain management strategies among women having reproductive surgeries. Fertil Steril. 2017;108(2):200-206. doi: 10.1016/j.fertnstert.2017.06.010

6. Zhang J, Zhou H, Sheng K, Tian T, Wu A. Foetal responses to dexmedetomidine in parturients undergoing caesarean section: a systematic review and meta-analysis. J Int Med Res. 2017;45(5):1613-1625. doi: $10.1177 / 0300060517707113$

7. Bellieni CV, Vannuccini S, Petraglia F. Is fetal analgesia necessary during prenatal surgery?. J Matern Fetal Neonatal Med. 2017;31(9):1241-1245. doi:
10.1080/14767058.2017.1311860

8. Phillips $\mathrm{CH}$, Wortman JR, Ginsburg ES, Sodickson AD, Doubilet PM, Khurana B. First-trimester emergencies: a radiologist's perspective. Emerg Radiol. 2018;25(1): 6172. doi: 10.1007/s10140-017-1556-9.

9. Leffert L, Butwick A, Carvalho B, Arendt K, Bates SM, Friedman A, et al. The society for obstetric anesthesia and perinatology consensus statement on the anesthetic management of pregnant and postpartum women receiving thromboprophylaxis or higher dose anticoagulants. Anesth Analg. 2018;126(3):928-944. doi: 10.1213/ ANE. 0000000000002530

10. Hata T. Current status of fetal neurodevelopmental assessment: Four-dimensional ultrasound study. J Obstet Gynaecol Res. 2016;42(10):1211-1221. doi: 10.1111/ jog.13099 